\title{
Plasmonic Sensor: Towards Parts-per-Billion Level Sensitivity
}

\author{
Yoshiaki Nishijima, ${ }^{*}$ Syunsuke Suda, Gediminas Seniutinas, ${ }^{1,2}$ \\ Armandas Balčytis, ${ }^{1,3}$ and Saulius Juodkazis ${ }^{1}$ \\ Department of Electrical and Computer Engineering, Graduate School of Engineering, \\ Yokohama National University, 79-5 Tokiwadai, Hodogaya-ku, Yokohama, Kanagawa 240-8501, Japan \\ ${ }^{1}$ Centre for Micro-Photonics, Faculty of Engineering and Industrial Sciences, \\ Swinburne University of Technology, Hawthorn, VIC 3122, Australia \\ ${ }^{2}$ Paul Scherrer Institute, Villigen CH-5232, Switzerland \\ ${ }^{3}$ Institute of Physics, Center for Physical Sciences and Technology, \\ 231 Savanoriu, Avenue, LT-02300 Vilnius, Lithuania \\ (Received February 14, 2017; accepted May 17, 2017)
}

Keywords: plasmon sensor, gas detection, surface-enhanced infrared absorption

We have developed a plasmonic gas sensing system using transmissive (metal hole array) and scattering (rough surface of Au film) plasmonic materials in an integrating sphere geometry. The combination of transmissive and reflective plasmonic materials significantly enhances the detection threshold by way of surface-enhanced infrared absorption. Here, we first investigate the individual sensing enhancement contributions for each of the plasmonic materials and reveal the path towards applications in high-sensitivity gas detection.

\section{Introduction}

Localized surface plasmon resonance is an optical phenomenon arising from the coupling between light and the free electron plasma in a metal $(\mathrm{Au}, \mathrm{Ag}$, and $\mathrm{Cu})$ when the resulting oscillations are confined on nano-microstructures..$^{(1-3)}$ When incident light is resonant with the plasmon modes of these structures, a strongly localized electromagnetic (EM) field is established (EM field enhancement), particularly at sharp corners, edges, gaps, and interfaces. This phenomenon is widely used for spectroscopic signal enhancement, namely, for surface-enhanced Raman scattering $(\text { SERS) })^{(4-6)}$ and for surface-enhanced infrared absorption (SEIRA)..$^{(4,5,7-11)}$ Such measurement techniques are promising for constructing sensor systems with sensitivity of down to a single molecule as well as high specificity, since they yield spectroscopic information enabling molecular fingerprinting. The peculiarities of these mechanisms have been widely reported. ${ }^{(12,13)}$ From established results, it appears that EM field enhancement plays a crucial role in increasing sensitivity, as a strong localized EM field enhances both the molecular scattering and the absorption cross sections. Therefore, recent SERS and SEIRA studies have been focused on the control of EM field intensity by well-defined nano-microstructures. Nanogap structures and bowtie antennas are among the most thoroughly investigated examples. Furthermore, previously, we showed that pattern randomization can further enhance EM localization for nanoparticles with slightly different sizes. Certainly, it is prescient to develop specific sensor components, such as metal patterns for a strong EM enhancement; however, constructing a complete sensing system is also very important and

*Corresponding author: e-mail: nishijima-yoshiaki-sp@ynu.ac.jp

http://dx.doi.org/10.18494/SAM.2017.1580 
can provide additional means to engineer and improve sensitivity. Particularly for detecting low concentrations of molecules, it is crucial to harness light-matter interactions to the highest possible extent. Recently, we have succeeded in constructing a non-dispersive infrared (NDIR) gas sensing system with the metal hole array (MHA) type of plasmonic mesh surfaces. ${ }^{(7,14)}$

By using a MHA as an optical window in the NDIR system chamber, more than 10 times higher sensitivity has been obtained. In a typical NDIR measurement, the optical path length must be increased to $20 \mathrm{~cm}$ or more owing to the low molecular absorption cross-section. Therefore, plasmonic enhancement provides a significant advantage for gas detection and miniaturization of the setup. Also, a spectral selectivity window can be established and matched to absorption bands particular to certain molecular species by modifying the periodicity of MHAs. However, further sensitivity enhancement when using only MHAs is limited. Multilayered MHAs could be used, although owing to their low transmissivity, the system would exhibit excessive optical losses. Therefore, costly components such as intense light sources and sensitive detectors would become a necessity. To sidestep these limitations, we combine transmissive and reflective plasmonic materials in a geometry that maximizes the optical path and the number of light-matter interaction events.

Integrating spheres, which are cavities with inner surfaces coated by highly reflective materials, can generate a homogeneous light source during a multitude of scattering events. They are often used for determining the absolute quantum efficiency of light absorption, luminescence, reflectance, and scattering. ${ }^{(15)}$ They can also be used for augmenting NDIR gas cells to increase the optical path length and thereby to miniaturize the sensing system. ${ }^{(16)}$ If the surface of the integrating sphere is covered with a scattering plasmonic material, enhancement of the SEIRA signal is expected as a result of multiple interaction events. In this study, we report a further improvement in sensitivity by integrating MHAs in an integrating sphere and experimentally verify the idea as a proof-of-concept.

\section{Samples and Methods}

In this study, ammonia gas was used as a target sensing material. The transmissive plasmonic metal hole arrays were fabricated using conventional photolithography techniques. ${ }^{(7)}$ A positive tone photoresist was coated on the surface of a $\mathrm{Si}$ substrate (low resistivity, both sides mirrorpolished). Specifically for ammonia detection, a hexagonal lattice MHA has been fabricated with 3.3 $\mu \mathrm{m}$ periods and $1.7 \mu \mathrm{m}$ hole diameter. The photomask was put in contact with the resist layer and exposed to UV light, followed by a subsequent development step in $2.38 \%$ tetramethylammonium hydroxide (TMAH). Metal patterns were then created by evaporating $2 \mathrm{~nm}$ of $\mathrm{Cr}$ and $50 \mathrm{~nm}$ of $\mathrm{Au}$ and performing a lift-off procedure in acetone followed by a methanol rinse. Transmission spectra of the MHA were recorded using Fourier transform infrared (FT-IR) spectroscopy (FTIR6200 , JASCO Corporation). The walls of the integrating sphere were coated by a rough layer of $\mathrm{Au}$, which was prepared by thermally evaporating Au onto a kapton film. The reflectance of the resulting surface was around $80 \%$.

The gas sensor system was constructed using an integrating sphere as a gas flow cell. A 100 ppm mixture of ammonia was further diluted with pure nitrogen in a gas mixer to control its concentration in the range from 10 to $100 \mathrm{ppm}$, then injected into the integrating sphere. The cavity multiplies the number of reflections and scattering events before light reaches the detector. Therefore, a multiplicative enhancement of the SEIRA signal was expected. 
Figure 1 shows a schematic illustration of the concept of an integrating sphere as a gas detection system. The radiation emitted from a tungsten thermal filament used as the infrared light source propagates into the integrating sphere either through a MHA or a plain Si window. After entering the optical cavity, the infrared light is multiply reflected by the scattering plasmonic materials lining the walls of the sphere. The right panel of Fig. 1 shows one hemisphere of the integrating cavity. The Au-coated kapton film was cut into measured strips, which were then glued onto the internal wall of the integrating sphere. As a control, the performance of the integrating sphere was compared with that of a $2 \mathrm{~cm}$ optical path gas cell without a reflective plasmonic material wall coating. After many interaction events, infrared light with the spectral imprint of enhanced absorption was registered using a pyroelectric element, whose output voltage was measured using a data logger. This infrared radiation detector, which has a faster response time and a higher sensitivity than thermopile, was situated in the off-axis output port, separated from the rest of the cavity by another MHA or plain Si window. MHAs at input and output ports act as both an infrared absorption enhancement surface as well as a gas-specific band-pass filter. In the case of ammonia, molecular rotational mode IR absorption peaks appear in the spectral range from 8.5 to $12 \mu \mathrm{m}$. Therefore, for further specificity, an additional $10 \pm 0.3 \mu \mathrm{m}$ band-pass filter was placed between the cavity and the detector.

\section{Results and Discussion}

Figure 2 shows an optical transmission spectrum of the $3.3 \mu \mathrm{m}$ periodicity and $1.7 \mu \mathrm{m}$ hole diameter MHA structure fabricated over the entire area of a $2 \times 2 \mathrm{~cm}^{2}$ Si substrate. A plasmonic transmission peak appeared at around $11 \mu \mathrm{m}$ wavelength, well-matched to ammonia, whose rotational modes span the 8.5 to $12 \mu \mathrm{m}$ range. ${ }^{(17,18)}$

Initial experiments involved checking whether plasmon resonance can enhance ammonia's IR absorptions. Previously, we demonstrated optical vibrational mode enhancement by the MHAs. ${ }^{(7,14)}$ However, it is unclear if MHA plasmon resonance would have a similar effect on the rotational absorption bands. For this purpose, a $2 \mathrm{~cm}$ optical path gas cell equipped with FT-IR spectroscopic detection was used. Rotational mode spectra show narrow symmetrical multiple peaks. Figure
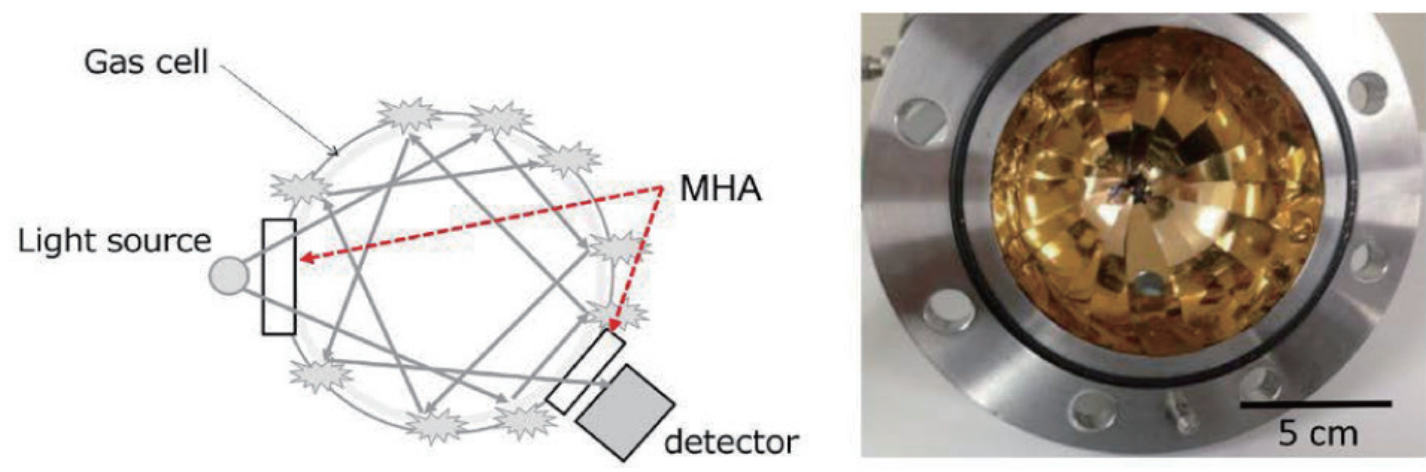

Fig. 1. (Color online) Schematic illustration of an integrating spherical gas cell. The inner wall of the gas cell is covered with a gold-coated kapton film, which acts as a scattering plasmonic material. 


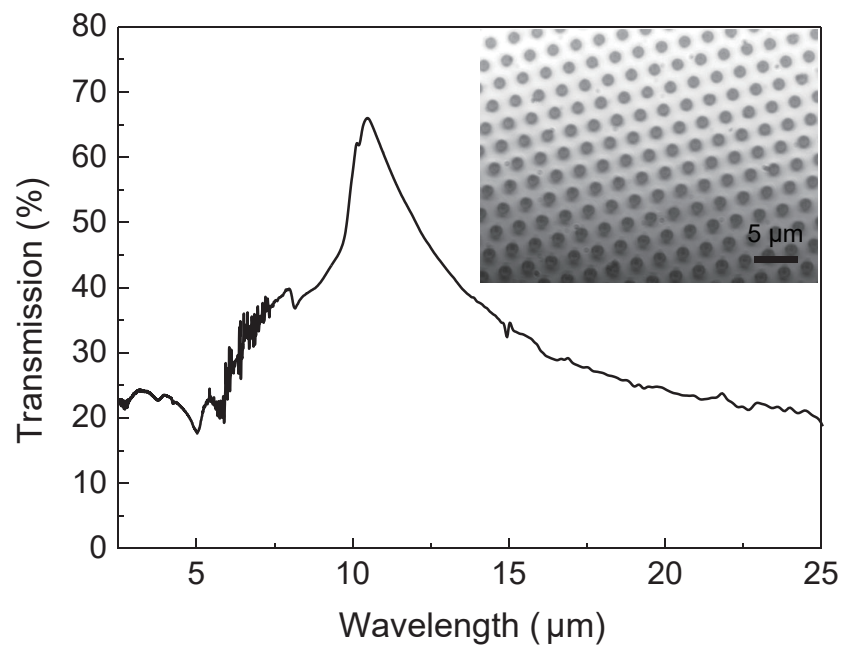

Fig. 2. Transmission spectrum of the MHA substrate with $3.3 \mu \mathrm{m}$ hole periods and $1.7 \mu \mathrm{m}$ hole diameters. The inset shows the optical reflection microscopy image of the MHA.

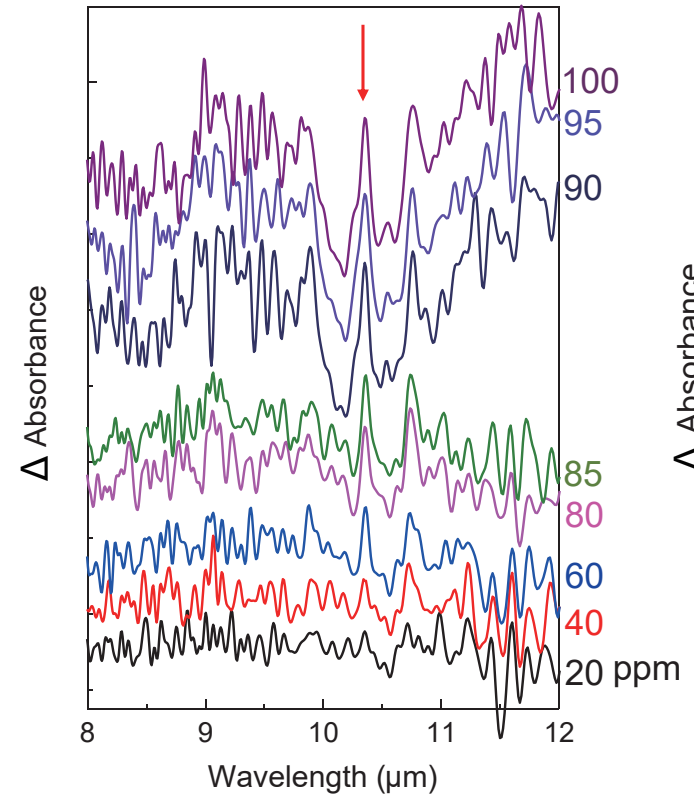

(a)

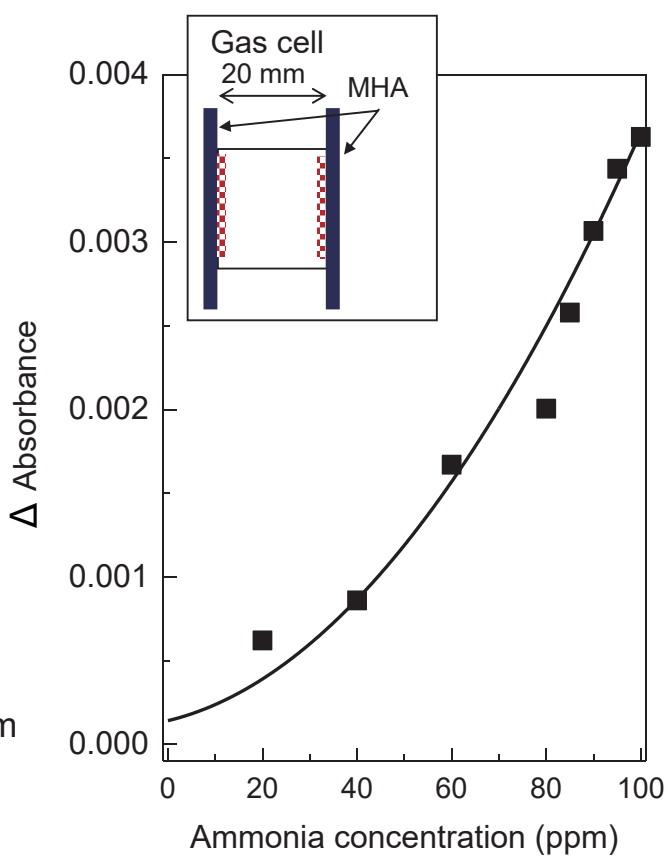

(b)

Fig. 3. (Color online) (a) Infrared absorbance spectra at different ammonia concentrations (from 20 to $100 \mathrm{ppm}$ ) obtained using MHA windows. (b) The dependence of the baseline adjusted at $10.5 \mu \mathrm{m}$ wavelength absorbance peak intensity ( $\triangle$ Absorbance) on ammonia concentration.

3(a) shows the ammonia-specific rotational peaks around a wavelength of $10.5 \mu \mathrm{m}$ scale with gas concentration from 20 to $100 \mathrm{ppm}$ when MHA optical windows are used. The baseline-adjusted $10.5 \mu \mathrm{m}$ peak absorption is plotted in Fig. 3(b). Considering the signal-to-noise ratio of the spectra, it is possible to reliably detect $60 \mathrm{ppm}$ concentrations of ammonia when MHAs are used. However, when no plasmonic MHA is present on the Si windows, no ammonia-specific absorption 
peaks were observed over the same concentration range. Therefore, we can conclude that plasmon resonance indeed enhances not only the vibrational but also the rotational modes owing to the localized EM field enhancement and spectral filtering of MHA.

Having confirmed the plasmonic IR absorption enhancement of the rotational modes, we exchanged the spectroscopic source and detector of the NDIR gas setup for the more straightforward thermal source and pyroelectric detector. ${ }^{(14)}$ Figure 4 shows the experimental results for ammonia gas sensing in the NDIR setup. Using the same $2 \mathrm{~cm}$ optical path length gas cell with two MHA windows employed in the FT-IR detection experiments, we could likewise detect the gas absorption signal at a concentration of around $60 \mathrm{ppm}$. However, as in the spectroscopic detection case, without MHA windows, no signal change was observed at or below $100 \mathrm{ppm}$.

When the gas cell was exchanged for the integrating sphere, even without MHA on any of the illumination and detection windows, it was possible to detect ammonia concentrations above the $50 \mathrm{ppm}$ level. This result indicates that metal films on the cavity walls can also enhance the IR absorption signals of ammonia gas by a combination of increased optical path and plasmon resonance effects. In addition to the use of the integrating sphere, MHAs were added as the light injection/extraction windows. The detection threshold for ammonia was markedly lowered to below $10 \mathrm{ppm}$. The combination of MHAs and reflective internal walls of the cavity strongly enhances the IR absorption.

The scattering/reflective materials lining the integrating sphere cavity had a reflectivity of $\sim 80 \%$. According to the integrating sphere theory, the throughput $T$ of the sphere can be expressed using the equation: ${ }^{(16,19,20)}$

$$
T=\frac{I_{\text {out }}}{I_{\text {in }}}=\frac{\rho A_{\text {out }}}{1-\rho\left(1-A_{\text {total }}\right)},
$$

where $I_{\text {in }}$ and $I_{\text {out }}$ are the input and output light intensities, $\rho$ is the reflectivity of the wall, and $A_{\text {out }}$ and $A_{\text {total }}$ are the output port area and total area of all ports, respectively, normalized to the

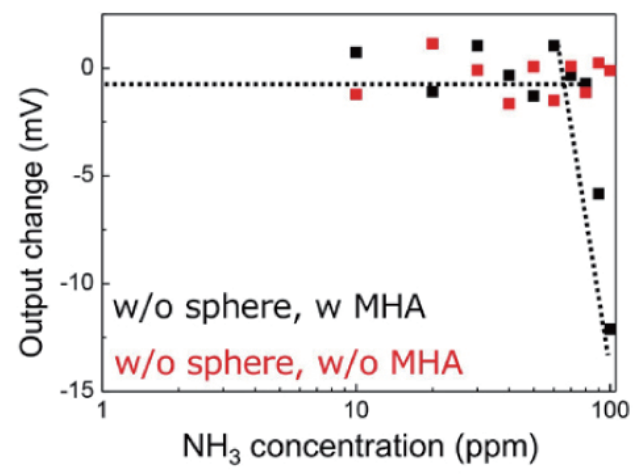

(a)

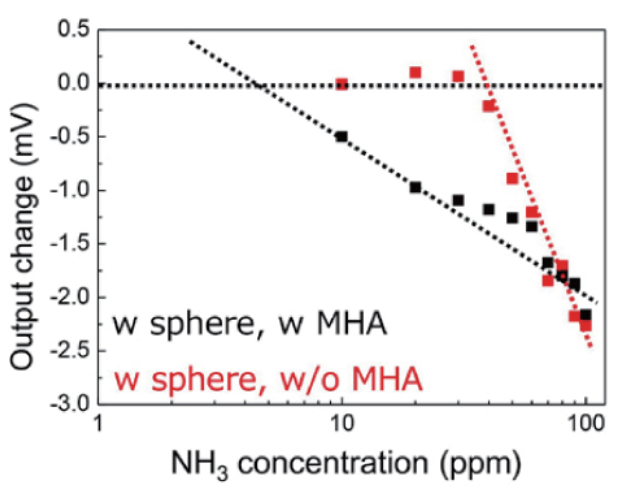

(b)

Fig. 4. (Color online) Ammonia gas detection results with (a) $2 \mathrm{~cm}$ optical path gas sensing system (without integration sphere) with MHA window (with MHA) and without MHA (without MHA), and (b) integrating sphere gas cell (with sphere) with/without MHA. 
entire surface area of the integrating sphere. In our system, $T$ is estimated to be around $2 \times 10^{-2}$, meaning that the expected average number of reflections is around 15 . These results were achieved without any specific optimization of plasmonic materials. If the SEIRA enhancement factor and the absolute reflectivity of the surface were increased, the detection thresholds would be improved toward ppb levels.

\section{Conclusion}

Sub-10 ppm ammonia gas detection thresholds using a combination of MHAs and an integrating sphere for absorption enhancement were demonstrated. Both the MHAs and the integrating sphere are two additive augmentations for gas sensors in NDIR. Improvement and optimization of the plasmonic materials such as MHAs together with scattering integrating sphere walls are promising future directions for ppm- and ppb-level detection setups, which can be both compact and exhibit high sensitivity/specificity. Such systems are required especially for biomedical applications in studies of exhaled air.

\section{Acknowledgments}

YN is grateful for partial support from the Japan Society for the Promotion of Science (JSPS), Grants-in-Aid for Scientific Research, Open Partnership Joint Projects of JSPS Bilateral Joint Research Projects, Tateishi Foundation, and the Amada Foundation. SJ is grateful for partial support via the Australian Research Council DP130101205 Discovery project.

\section{References}

1 Y. Nishijima, L. Rosa, and S. Juodkazis: Opt. Express 20 (2012) 11466.

2 Y. Nishijima and S. Akiyama: Opt. Mater. Express 2 (2012) 1226.

3 Y. Hashimoto, G. Seniutinas, A. Balcytis, S. Juodkazis, and Y Nishijima: Sci. Rep. 6 (2016) 25010.

4 Y. Nishijima, Y. Hashimoto, L. Rosa, J. B. Khurgin, and S. Juodkazis: Adv. Opt. Mater. 2 (2014) 382.

5 Y. Nishijima, J. B. Khurgin, L. Rosa, H. Fujiwara, and S. Juodkazis: ACS Photonics 1 (2014) 1006.

6 H. Wang, J. Kundu, and N. J. Halas: Angew. Chem. 46 (2007) 9040.

7 Y. Nishijima, H. Nigorinuma, L. Rosa, and S. Juodkazis: Opt. Mater. Express 2 (2012) 1367.

8 B. Cerjan, X. Yang, P. Nordlander, and N. J. Halas: ACS Photonics 3 (2016) 354.

9 Y. Sato, H. Noda, F. Mizutani, A. Yamakata, and M. Osawa: Anal. Chem. 76 (2004) 5564.

10 H. Aouani, H. Sipoca, M. Rahmani, M. N. Cia, K. Hegnerva, J. Homola, M. Hong, and S. A. Maier: ACS Nano 7 (2013) 669.

11 T. Neuman, C. Huck, J. Vogt, F. Neubrech, R. Hillenbrand, J. Aizpurua, and A. Pucci: J. Phys. Chem. C 119 (2015) 26652.

12 J. S. Hartley, M. M. Hlaing, G. Seniutinas, S. Juodkazis, and P. R. Stoddart: Biomicrofluidics 9 (2015) 061101.

13 G. Seniutinas, G. Gervinskas, R. Verma, B. D. Gupta, F. Lapierre, P. R. Stoddart, F. Clark, S. L. McArthur, and S. Juodkazis: Opt. Express 23 (2015) 6763.

14 Y. Nishijima, Y. Adachi, L. Rosa, and S. Juodkazis: Opt. Mater. Express 3 (2013) 968.

15 Y. Nishijima, R. Komatsu, S. Ota, G. Seniutinas, A. Balcytis, and S. Juodkazis: APL Photonics 1 (2016) 076104.

16 J. A. Jacquez and H. F. Kuenheim: J. Opt. Soc. Am. 45 (1955) 460.

17 D. M. Dennison and J. D. Hardy: Phys. Rev. 39 (1932) 938.

18 W. L. France and D. Williams: J. Opt. Soc. Am. 56 (1966) 70.

19 P. Chambers, W. B. Lyons, T. Sun, and K. T. V. Grattan: Sens. Actuators, A 162 (2010) 20.

20 M. W. Finkel: Opt. Commun. 2 (1970) 25. 